\title{
Research on Single Drive and Multi-dimension Output Vibrator for Fuse Sand Filling Based on Decoupled 1T2R Parallel Mechanism
}

\author{
Qin Wei, Hang Lubin, Cheng Wushan and Huangfu Yabo \\ College of Mechanical Engineering, Shanghai University of Engineering Science, China
}

\begin{abstract}
In this paper a three-dimension vibrator for fuses' sand filling, including one-dimension vertically translation and two-dimension oscillating rotation (1T2R) is presented, which is used to improve the low efficiency in the process of traditional fuses' sand filling. The paper starts with a type synthesis of a new kind of 1T2R parallel mechanism (PM) which is constructed based on the POC set. After two of the driving joints in the PM are substituted by the spring-damp system, the design propose of single-input and multi-outputs can be realized. Then the kinematics analysis and simulation optimization of this mechanism was also carried out.
\end{abstract}

\section{Introduction}

Fuse is an important component of the smart grid user end system, some problems still remains in the production process of the fuse [1]. Since the fuse can improve the electrical stability and thermal stability of the electrical appliances [2], [3] and the density of quartz sand filled in the fuse box has a direct and significant influence on the breaking performance of the fuse [4]-[6].

Single-dimension sand filling vibration appliances [7], shown in Fig. 1. (a) (b), are widely used in domestic low voltage electrical enterprises. As is shown in Fig. 1.(c) The fuse sand filling unit consists of flexible sand filling nozzle 1 , fuse 2 , electromagnetic coil components 3 , spring 4 , base 5 .

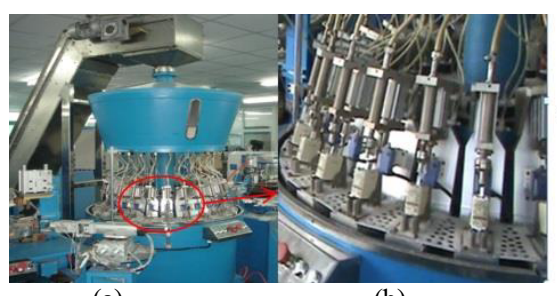

(a)

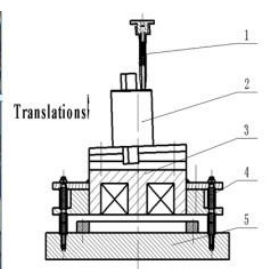

(c)
Figure 1. Mechanical fuses sand filling device

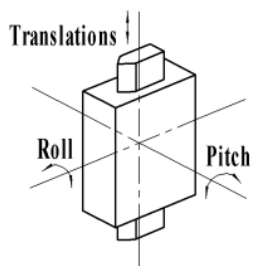

Figure 2. Expected motion of fuse in the process of sand filling

For the reasons that the traditional single-dimension vertical vibrator is difficult to meet the requirements of sand filling density. The paper proposed a novel three- dimension vibrator for fuses' sand filling, including onedimension vertical translation and two-dimension rotations (shown in Fig. 2), based on POC set [8].

In comparison to the traditional fuse's sand filling vibrator, the two additional rotary vibration of the new designed PM exerted on the fuse can make the fuse get oscillated in roll and pitch directions, which can spread the quartz sands in the fuse uniformly, compact the finegrained quartz sands and improve the density of fuse product.

The paper is organized as follows. The type synthesis of 1T2R PM is given in section 2. The kinematic analysis is carried out in section 3 . The vibration simulation and geometry optimization of the 1T2R PM vibrator is carried out in section 4. Finally, conclusions are drawn in section 5 .

\section{Type synthesis of PM}

The multi-dimension fuse vibration platform can improve the density of quartz sand filled in the fuse. The platform needs to achieve a motion of one translation and two rotations, shown in Fig. 2. The input-output characteristics of mechanism should meet the requirement of the decoupled judgment criteria, the POC set of the decoupled PM can be derived as $M_{P_{a}}=\left[\begin{array}{l}t^{1} \\ r^{2}\end{array}\right]$.

\subsection{The construction of decoupled PM}

For the sake of reducing the control difficulty of the PM, the decoupled property should be combined with the mechanism in the design process.

According to the decoupled judgment criteria of the PM proposed by author of paper [9], the 1T2R PM shown 
in Fig. 3 is selected as the prototype to construct the multi-dimension vibrator because of its simplicity, and the structure synthesis and kinematics analysis are given in detail thereinafter, it consists of two basic kinematic chains (BKC). One of the loops is a $\mathrm{BKC}$ of planar fivebar loop with two driving translation joints $P_{1}, P_{2}$ and three revolute joints $R_{1}, R_{2}, R_{3}$, it can be denoted by $H S O C_{1}\left\{-P_{1} \perp R_{1}\left\|R_{2}\right\| R_{4} \perp P_{2}-\right\}$. The other loop is a $\mathrm{BKC}$ consist of a driving joints $P_{3}$, two spherical joints $S_{1}, S_{2}$ and a revolute joint $R_{2}$, it can be denoted by $S O C_{2}\left\{-P_{3}-S_{1}\right.$ $\left.S_{2}-R_{2}-\right\}$. The linkage $S_{2} R_{2}$ is the output moving platform, as is shown in Fig. 3.

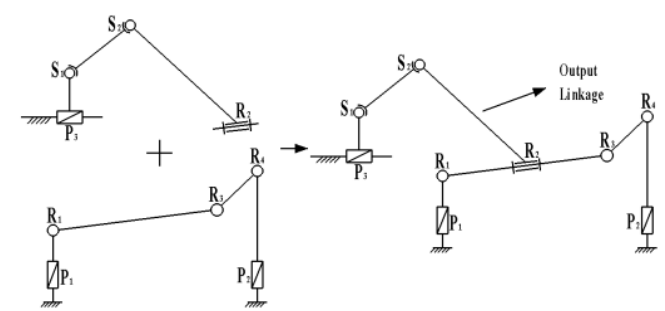

Figure 3. The construction of 1T2R PM

\subsection{PM synthesis with the output motion characteristic matrix}

1) POC matrix of each branch end

The POC matrix of the branch end of $H S O C_{1}$ and $S O C_{2}$ can be expressed as: $M_{b_{1}}=\left[\begin{array}{c}t^{1}(\| z) \\ r^{2}(\| \nabla(x, y))\end{array}\right], M_{b_{2}}=\left[\begin{array}{c}t^{3} \\ r^{3}\end{array}\right]$, respectively.

2) POC matrix of the moving platform of the PM

Substitute the POC matrix of the terminal branches of chain 1, 2 into formula $M_{p_{a}}=\bigcap_{i=1}^{v+1} M_{b_{i}}$ [10], the POC matrix of the moving platform is obtained.

$$
M_{p_{a}}=\left[\begin{array}{c}
t^{1}(\| z) \\
r^{2}(\| \nabla(x, y))
\end{array}\right] \bigcap\left[\begin{array}{l}
t^{3} \\
r^{3}
\end{array}\right]=\left[\begin{array}{c}
t^{1} \\
r^{2}
\end{array}\right]
$$

\subsection{The DOF of the mechanism}

The POC set of the moving platform is affected by the degrees of freedom (DOF) of the mechanism, so it's necessary to analyze whether the DOF of the PM can meet the demand of the POC set. According to the formula of DOF [11], we will obtain:

$$
F=\sum_{i=1}^{m} f_{i}-\sum_{j=1}^{v} \xi_{L_{j}}=12-(6+3)=3
$$

Where $m$-- the number of kinematic pairs

$f_{\mathrm{i}^{-}-}$the DOF of the $\mathrm{i}$ th kinematic pair

$v$-- the number of the independent loops

$\xi_{L^{-}-}$the number of the independent loop of the $\mathrm{j}$ th basic loop.

\section{Forward kinematics of 1T2R PM}

The static and moving coordinate systems are established as shown in Fig. 4. The angle between the y-axis and the $\mathrm{Y}$-axis is donated by $\alpha$, The angle between the $x$-axis and the $X$-axis is donated by $\beta$, the angle between the $\mathrm{X}$-axis and linkage $R_{3} R_{4}$ is denoted by $\gamma$, The longitudinal coordinate of the moving platform's center is denoted by $z$. the displacement of shifting pair $P_{\mathrm{i}}(\mathrm{i}=1,2,3)$ is denoted by $d_{\mathrm{i}}(\mathrm{i}=1,2,3)$, the distance of each two adjacent joints is denoted by $L_{\mathrm{i}}(\mathrm{i}=1,2 \ldots 9)$.

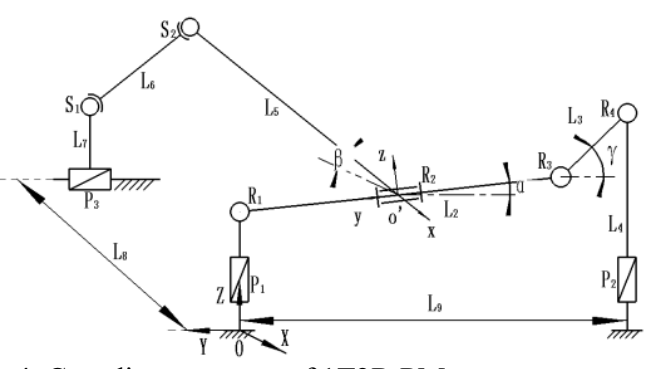

Figure 4. Coordinate system of 1T2R PM

\subsection{The governing kinematics equation of the PM}

Project the linkages in loop $-P_{1} \perp R_{1}\left\|R_{3}\right\| R_{4} \perp P_{2}$ - to the $X$ axis and the $Y$-axis respectively, and the projection equation of the loop will be obtained:

$$
\left\{\begin{array}{c}
L_{2} \cos \alpha+L_{3} \cos \gamma=L_{9} \\
L_{1}+d_{1}+L_{2} \sin \alpha+L_{3} \sin \gamma=L_{4}+d_{2}
\end{array}\right.
$$

Since the coordinates of spherical joints $S_{1}, S_{2}$ must satisfy the geometry constraint equation below [12], [13]:

$$
\left(X_{S_{2}}-X_{S_{1}}\right)^{2}+\left(Y_{S_{2}}-Y_{S_{1}}\right)^{2}+\left(Z_{S_{2}}-Z_{S_{1}}\right)^{2}=L_{5}^{2}
$$

So, the simultaneous equations of the mechanism kinematics can be expressed as:

$$
\left\{\begin{array}{c}
L_{2} \cos \alpha+L_{3} \cos \gamma=L_{9} \\
L_{1}+d_{1}+L_{2} \sin \alpha+L_{3} \sin \gamma=L_{4}+d_{2} \\
\left(X_{S_{2}}-X_{S_{1}}\right)^{2}+\left(Y_{S_{2}}-Y_{S_{1}}\right)^{2}+\left(Z_{S_{2}}-Z_{S_{1}}\right)^{2}=L_{5}^{2}
\end{array}\right.
$$

\subsection{Forward position problem}

The purpose of the forward position problem is to obtain the output parameters, i.e., the position parameters $z, \alpha, \beta$ of the moving platform's center, and the displacement $d_{1}$, $d_{2}, d_{3}$ of the shifting pairs $P_{1}, P_{2}, P_{3}$ are known.

$$
z=L_{1}+d_{1}+L_{2} \sin \alpha=L_{1}+d_{1}+L_{2} \sin \left(2 \arctan \frac{A \pm \sqrt{A^{2}+B^{2}-C^{2}}}{B-C}\right)
$$

From Eq. (5), two linear equation in variables $\alpha, \beta$ are obtained, expressed in the following way:

$$
\begin{aligned}
A \sin \alpha+B \cos \alpha+C & =0 \\
D \sin \beta+E \cos \beta+F & =0
\end{aligned}
$$

From Eq. (7) and Eq. (8), we can obtain:

$$
\begin{aligned}
& \alpha=2 \arctan \frac{A \pm \sqrt{A^{2}+B^{2}-C^{2}}}{B-C} \\
& \beta=2 \arctan \frac{D \pm \sqrt{D^{2}+E^{2}-F^{2}}}{E-F}
\end{aligned}
$$


where

$$
\begin{aligned}
& A=2 L_{2}\left(L_{4}+d_{2}-L_{1}-d_{1}\right) \\
& B=2 L_{2} L_{9} \\
& C=L_{2}^{2}-L_{3}^{2}-\left(L_{1}+d_{1}-L_{4}-d_{2}\right)^{2}-\left(L_{9}+d_{1}+d_{2}\right)^{2} \\
& D=\left(L_{9}+2 d_{3}+3 L_{2} \cos \alpha\right) L_{5} \sin \alpha+2\left(L_{1}+d_{1}-L_{7}\right) L_{5} \cos \alpha \\
& E=-2 L_{5} L_{8} \\
& F=-L_{6}^{2}+L_{8}^{2}+\left(\frac{1}{2} L_{9}+d_{3}+\frac{1}{2} L_{2} \cos \alpha\right)^{2}+\left(L_{1}+d_{1}+L_{2} \sin \alpha-L_{7}\right)^{2}
\end{aligned}
$$

\subsection{The reduction and selection of the driving pair}

For the reasons that the number of the vibration generators have an important influence on fuse vibration's cost, we propose to reduce the number of the driving joints to one. Since the loop 1 has partly symmetry in the structure, the active driving joints in loop 1 are substituted by the force adaptable elastic element to realize the design purpose of single-input and multi-outputs, that is to say the driving pairs $\mathrm{P}_{1}, \mathrm{P}_{2}$ are constrained by the spring-damp system based on the mechanism decoupled principles, and the loop 1 becomes the force adaptable servo loop. Since only one driver is needed in this mechanism, the operation cost of the fuse vibration is reduced. The shifting pair $\mathrm{P}_{3}$ is selected to be the vibration generator and the structure of the PM fuse vibrator is shown in Fig. 5.(a), and by the usage of the 24 potentiometers, which are shown in Fig. 5.(b), the vibration mode (working amplitude, pitch angle and roll angle) of the PM can be adjusted easily.

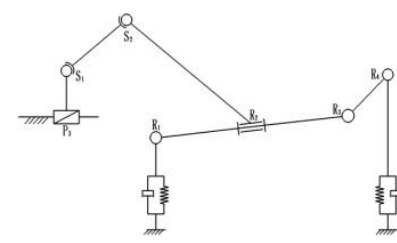

(a)

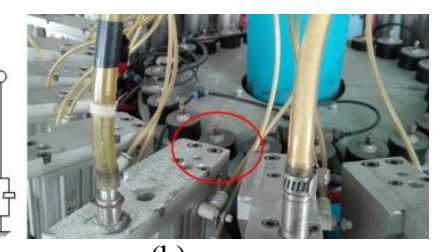

(b)
Figure 5. The mechanism sketch of 1T2R PM vibrator constrained by the spring-damp system and the potentiometers

\section{The simulation and optimization of the vibrator}

Experiment shows that the fuse vibrator with a working amplitude of $1 \sim 2 \mathrm{~mm}$, a pitch angle of $\pm 2^{\circ}$, a roll angle of $\pm 1^{\circ}$ can improve the fuse's sand filling density and the efficiency according to process parameters.

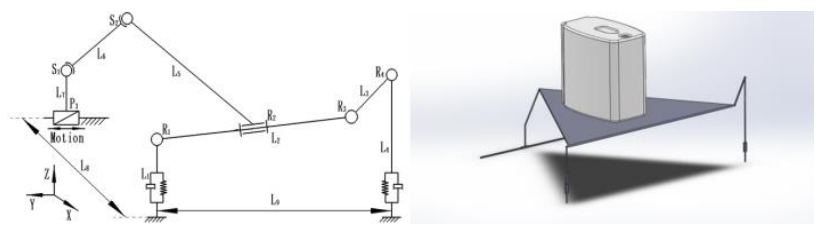

Figure 6. The mechanism sketch and $3 \mathrm{D}$ graph of the 1T2R PM

\subsection{The initial setup of the PM vibrator's size}

The simulation model of the PM is established in Solidworks, as shown in Fig. 6, and the size of the mechanism is set up according to the structure and the dynamic parameters of the vibrator [14]. The PM's structure parameters are set as: $\mathrm{L}_{1}=30 \mathrm{~mm}, \mathrm{~L}_{2}=220 \mathrm{~mm}$, $\mathrm{L}_{3}=22 \mathrm{~mm}, \mathrm{~L}_{4}=40.5 \mathrm{~mm}, \mathrm{~L}_{5}=84 \mathrm{~mm}, \mathrm{~L}_{6}=22 \mathrm{~mm}, \mathrm{~L}_{7}=11 \mathrm{~mm}$, $\mathrm{L}_{8}=100 \mathrm{~mm}$. The stiffness of the springs between the shifting pairs $\mathrm{P}_{1}, \mathrm{P}_{2}$ is $5 \mathrm{~N} / \mathrm{mm}$, and the damping coefficient is 0.035 .

\subsection{Dynamic simulation analysis}

In order to test the motion performance of the 1T2R PM vibrator we designed, the motion simulation model of the vibrator is established in ADAMS, the kinematic pairs are constrained, and the fuse model is added onto the center of the moving platform. The final simulation model is shown as Fig. 7.

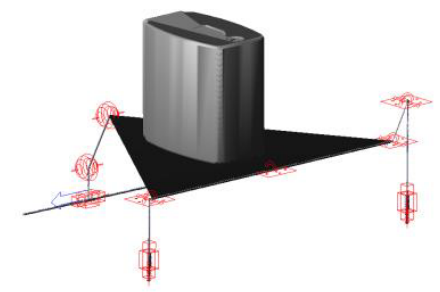

Figure 7. The structural diagram of the vibrator in ADAMS

According to the analysis of the type synthesis of the mechanism, the prismatic pair $\mathrm{P}_{3}$ is chose to be the driving pair, and the driving force is added to it to generate the vibration, the expression is: $\mathrm{d} 3=10 \sin (100 \pi \mathrm{t})$ $\mathrm{mm}, 50 \mathrm{~Hz}$. The simulation results (the working amplitude, the pitch angle and the roll angle) of the moving platform are shown as Figure 8-10.

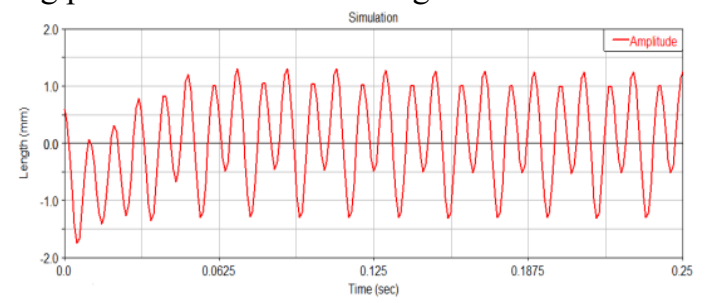

Figure 8. Simulation curve of fuse vibrator's working amplitude

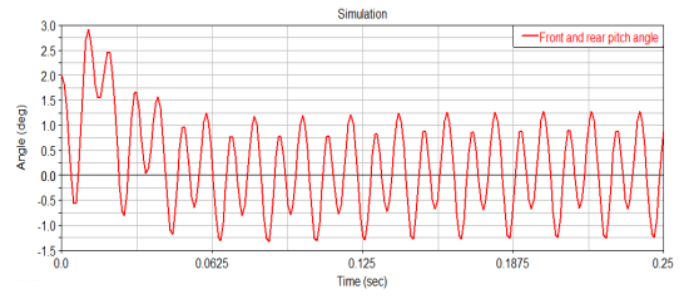

Figure 9. Simulation curve of fuse vibrator's pitch angle

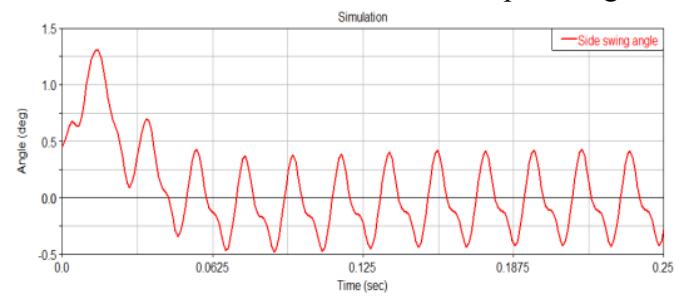

Figure 10. Simulation curve of fuse vibrator's roll angle

From the simulation curves above, we can see that the PM vibrator comes to a steady state after $0.0625 \mathrm{~s}$, the working amplitude and the pitch angle of the fuse 
vibration can meet the production index of mechanical sand filling, but the roll angle of the vibration is just $\pm 0.43^{\circ}$, and can't meet the working demand of $\pm 1^{\circ}$.

\subsection{Model parameter optimization}

The model parameter optimization must be carried out to make the roll angle of the vibration meet the production demand of mechanical sand filling as well.

By parametrical setting of each point in ADAMS, the influence of each design variables on the roll angle of the vibration can be analyzed, and we found that the $Z$ coordinate of the spherical pair $\mathrm{S} 1$, the $\mathrm{Z}$ coordinate of the spherical pair $S 2$ and the $Z$ coordinate of revolute joint has a great influence on the roll angle of the vibration.

Then the coordinates of the three reference points are adjusted to obtain the roll angle of the vibration. By using the ADAMS to optimize the three design variables, the coordinates of the design variables and the corresponding roll angle are optimized. The optimization results are shown in Table I.

Table 1. The optimization of the roll angle

\begin{tabular}{|c|c|c|c|c|}
\hline & Roll angle & $S_{1 Z}$ & $S_{2 Z}$ & $R_{2 Z}$ \\
\hline Initial Value & $\pm 0.4398^{\circ}$ & 11.000 & 27.000 & 30.250 \\
\hline Optimization & $\pm 0.8503^{\circ}$ & 11.007 & 25.472 & 30.599 \\
\hline
\end{tabular}

To sum up, the optimized size of the 1T2R PM fuse vibrator, which meet the demand of the mechanical sand filling working index can be set as: $\mathrm{L}_{1}=30 \mathrm{~mm}$; $\mathrm{L}_{2}=220 \mathrm{~mm} ; \quad \mathrm{L}_{3}=22 \mathrm{~mm} ; \mathrm{L}_{4}=40.5 \mathrm{~mm} ; \mathrm{L}_{5}=89.629 \mathrm{~mm}$; $\mathrm{L}_{6}=22.572 \mathrm{~mm} ; \mathrm{L}_{7}=11.258 \mathrm{~mm} ; \mathrm{L}_{8}=100 \mathrm{~mm}$.

\section{Conclusion}

(1) To improve the efficiency of the traditional single dimensional vertical sand filling vibrator, a threedimension vibrator based on the decoupled PM for fuses' sand filling is presented.

(2) The decoupled 1T2R PM is established based on the POC set and the decoupled criterion.

(3) To reduce the number of the driving joints of the PM, a creative method of substituting the two prismatic pairs in loop 1 by spring-damp system is proposed. The mechanism can provide one vertical translation and two rotations output motion in the condition of one input motion.
(4) The motion simulation of the mechanism is carried out in ADAMS, and roll angle of the fuse vibration is optimized by using the principle of sensitive point.

\section{Acknowledgement}

The authors would like to acknowledge the financial support of the national science foundation of China (NSFC) with grant number of 51475050, Shanghai Science and Technology Committee with grant number of 12510501100, the Natural Science Foundation of Shanghai City with grant number of 14ZR1422700, and the Graduate student research Innovation Project of Shanghai University of Engineering Science (SUES) with grant number of E109031501012 and E109031501016.

\section{References}

1. X.M. Wang. Electric Power Technologic and Economics. 22, 1-7 (2010).

2. M.A. Saqib, AD Stokes. IEEE Trans. Power. Del. 25, 212-220 (2010).

3. A. Pleşca, INT J THERM SCI. 70, 144-153 (2013)

4. X.Q. Chen, H.P. Fan, J.M. Wang. Low Voltage Apparatus. 01,44-47 (1986).

5. W. Bussiere. J. Phys. D: Appl. Phys. 34, 925 (2001)

6. Y.Y. Xiao, J.W. Zhuang, B Chen. J. Harbin Eng. Univ. 34, 524-529 (2013).

7. Zhang Minliang, Cheng Wushan, Lin Haiou, Hang Lubin, Yang Huibin, Yue Min. A kind of intermittent rotary continuous circulating type fuse sand filling machine[P]. Shanghai: CN102915881A, 2013-02-06

8. T.L. Yang. Theory and Application of Robot Mechanism Topology, Beijing Science Press, (2012).

9. L.B. Hang, Y Wang, T.L. Yang. China Mechanical Engineering. 15, 1035-1037,1041, (2004).

10. T.L. Yang, A.X. Liu, Q Jin, et al. J. Mech. Des. 131, 021001-1-17, (2009).

11. T.L. Yang, DJ Sun. J. Mechanisms Robotics. 4, 011001-1-17, (2012).

12. J J. Craig Introduction to robotics: mechanics and control, Upper Saddle River: Pearson Prentice Hall, (2005).

13. Y Yun, Y Li. J. Dyn. Sys. Meas. Control. 134, 021001-1-9, (2012).

14. B Li, W Zhao, Z Deng. JMSY. 31, 50-58, (2012). 